\title{
Profiling microRNAs in Eucalyptus grandis reveals no mutual relationship between alterations in miR156 and miR172 expression and adventitious root induction during development
}

\author{
Aviv Levy 1,2, David Szwerdszarff,2,3, Mohamad Abu-Abied', Inna Mordehaev', Yossi Yaniv', Joseph Riov², \\ Tzahi Arazi ${ }^{1}$ and Einat Sadot ${ }^{1 *}$
}

\begin{abstract}
Background: The change from juvenile to mature phase in woody plants is often accompanied by a gradual loss of rooting ability, as well as by reduced microRNA (miR) 156 and increased miR172 expression.

Results: We characterized the population of miRNAs of Eucalyptus grandis and compared the gradual reduction in miR156 and increase in miR172 expression during development to the loss of rooting ability. Forty known and eight novel miRNAs were discovered and their predicted targets are listed. The expression pattern of nine miRNAs was determined during adventitious root formation in juvenile and mature cuttings. While the expression levels of miR156 and miR172 were inverse in juvenile and mature tissues, no mutual relationship was found between high miR156 expression and rooting ability, or high miR172 expression and loss of rooting ability. This is shown both in E. grandis and in E. brachyphylla, in which explants that underwent rejuvenation in tissue culture conditions were also examined.
\end{abstract}

Conclusions: It is suggested that in these Eucalyptus species, there is no correlation between the switch of miR156 with miR172 expression in the stems and the loss of rooting ability.

Keywords: miRNA, Eucalyptus, Juvenile to mature phase change, Adventitious roots

\section{Background}

Plant species gradually mature through four phases: (1) embryonic, (2) postembryonic juvenile vegetative, (3) mature vegetative, and (4) mature reproductive [1,2]. The most pronounced maturity trait is flowering capacity, but other traits, such the ability to form adventitious roots (ARs), are modified during the phase changes [2-6]. In many woody plants, rooting ability is expressed at the base of the stem, which retains juvenile characteristics throughout the plant life, and is sharply or gradually lost upon transition to the mature phase [2].

Initial indications of the molecular control of the vegetative phase change (phase 2 to 3 above) came from

\footnotetext{
*Correspondence: vhesadot@agri.gov.il

${ }^{1}$ The Institute of Plant Sciences, Volcani Center, P.O. Box 6, Bet-Dagan 5025000, Israel

Full list of author information is available at the end of the article
}

analyses of maize (Zea mays) mutants. The Teopod1 and Teopod 2 mutations led to extended expression of juvenile traits, among which was an increase in the number of nodes bearing ARs [7]. In contrast, the Glossy15 mutation in maize accelerated the appearance of adult characteristics in juvenile leaves [8,9]. Later, the Glossy15 gene, that encodes APETALA2-like [10], was found to be regulated by miR172 [11,12]. Corngrass $1(C g 1)$ is a maize mutant in which juvenile traits are retained during the adult reproductive phase [13]. The molecular basis of Cg1 is overexpression of miR156, concomitant with lower levels of miR172 [13]. Similarly, tomato (Solanum lycopersicum cv. 'Ailsa Craig') plants overexpressing miR156 exhibited increased development of stem ARs [14]. MiR156 and miR172 have been found to coordinate the juvenile-to-mature phase change in Arabidopsis. miR156 targets the transcription factors SQUAMOSA 
PROMOTER BINDING PROTEIN LIKE (SPL) 9 and 10 that promote miR172b transcription, which in turn exhibits a regulatory feedback loop with its targets TOE1 and 2 [12].

miR156 and miR172 have been shown to have similar expression patterns in various woody plants, including Acacia confusa, Acacia colei, Eucalyptus globulus, Hedera helix, Quercus acutissima, and Populus x canadensis, during the juvenile-to-mature phase change [15]. Overexpression of miR156 in transgenic poplar (Populus $\mathrm{x}$ canadensis) reduced the expression of miR156-targeted SPL genes and miR172 and prolonged the juvenile phase [15]. Taken together, these data indicate that miR156 is an evolutionarily conserved regulator of the vegetative phase change in both herbaceous and woody plants, whereas miR172 is a regulator of maturity [12,15].

Other miRNAs have been shown to be directly involved in AR formation in Arabidopsis, with ARF17, a target of miR160, being a negative regulator, and ARF6 and $A R F 8$, targets of miR167, being positive regulators [16]. These transcription factors are also involved in regulation of the cross-talk between auxin and jasmonic acid pathways during AR formation [17].

The juvenile-to-mature phase change of Eucalyptus grandis was previously characterized [18]. It was shown that rooting capability was higher in cuttings excised below node 5 , termed juvenile, than in those excised above node 15, termed mature [18]. The sequencing of Eucalyptus grandis genome has made the plant a valuable experimental system. In the present study, a comprehensive profiling of miRNAs was carried out and the expression of miR156 and miR172 was studied in relation to the loss of rooting ability in juvenile and mature cuttings of E. grandis and E. brachyphylla.

\section{Results}

\section{Profiling of miRNA expression}

To obtain a maximum spectrum of miRNAs expressed in E. grandis, total RNA was extracted from 14-day-old intact seedlings and subjected to miRNA discoveryoriented deep sequencing. After filtering, a large number of the reads matched t/rRNA sequences (Table 1). Analysis by the ShortStack application [19] revealed 40 known and 8 novel microRNAs in the E. grandis RNA

Table 1 Sequence data analysis

\begin{tabular}{lll}
\hline & Total & Non redundant \\
\hline Input & $6,989,302$ & 72,258 \\
Valid sequence filter & $6,891,830$ & 67,710 \\
t/rRNA filter & $2,111,579$ & 49,097 \\
Genome filter & $1,273,259$ & 32,300 \\
\hline
\end{tabular}

A series of digital filters were employed on the raw readings to discard sequences irrelevant to microRNA identification. sample (Additional file 1: Table S1). Among the conserved microRNAs were miR156, miR157, miR160, miR164, miR166, miR167, miR171, miR172, miR319, miR390, miR394, miR395, miR396, miR408, and miR828. BLAST results for the predicted targets of conserved miRNAs identified in E. grandis (Additional file 2: Table S2) indicated that most of them were similar to predicted targets documented in other plants. The predicted targets for the novel miRNAs identified by the ShortStack analysis are listed in Additional file 3: Table S3.

Northern blot analysis was performed to determine possible changes in miRNA expression during induction of AR formation in juvenile and mature cuttings (Additional file 4: Figure S1). Cuttings were excised from different stem sections of stock plants of the same chronological age. Juvenile cuttings were excised from 10 to $15 \mathrm{~cm}$ above the ground, and mature cuttings from about $2 \mathrm{~m}$ above the ground. Under these experimental conditions, AR primordia start to form, after 3 to 9 days only in juvenile cuttings, confirming earlier studies showing that shoots in close proximity to the root system retain juvenile traits [18]. No consistent differences were found between juvenile and mature cuttings in the expression of miR160, 164, 166, 167, 171, 396 and 397, during the first 9 days after excision and auxin application. In addition, the expression levels of the corresponding predicted targets of these miRNAs were analyzed in the above cuttings by the nanostring method (Additional file 5: Table S4). No consistent differences were found between them, except for NAC1 which was gradually upregulated only in juvenile cuttings during $A R$ induction (Additional file 5: Table S4).

\section{Expression of miR156 and miR172 in relation to the loss of rooting ability}

Changes in miR156 and miR172 expression have been found to be key regulators of the juvenile-to-mature phase change [12]. Here, the pattern of miR156 and miR172 expression was determined in the tissue giving rise to AR formation in two plant systems. The first was E. grandis, in which a third type of cutting was excised $45 \mathrm{~cm}$ above the ground, termed midphase (Figure 1a). The rooting potential was found to decrease with increasing distance from the ground from which the cuttings were collected (Figure 1b). In parallel to the loss of rooting potential, an increase in callus formation was observed, in agreement with previous findings in other woody plants [20-22]. While miR156 expression declined slightly during maturation, miR172 exhibited an opposite pattern, with significant elevated levels in mature cuttings compared to juvenile ones (Figure 1c,d), in agreement with previous findings in other trees [15]. Interestingly, in midephase, the decline in expression of miR156 and the increase in 


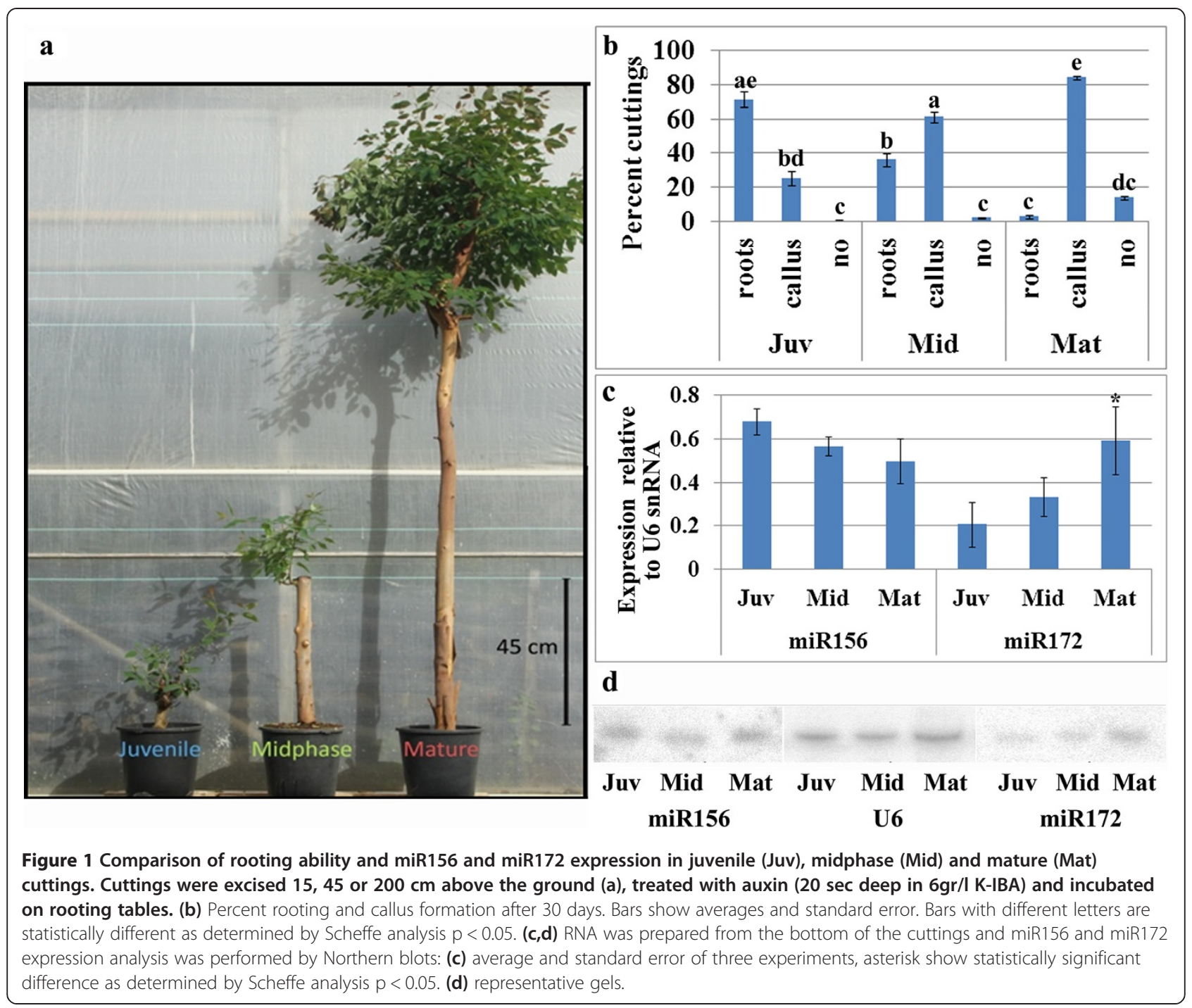

that of miR172 compared to juvenile cuttings was not significant, but the difference in rooting ability and increase in callus formation between these cutting was statistically significant (Figure 1b). We concluded that the loss of rooting ability in E. grandis may precede a significant decline and increase in miR156 and miR172 expression, respectively, in the tissue giving rise to AR formation.

\section{Expression of miR156 and miR172 in relation to loss of rooting ability in E. brachyphylla}

We examined the relationship between miR156 and miR172 expression and the loss of rooting ability in another Eucalyptus species, E. brachyphylla. This species is an ornamental plant characterized by bluish leaves. Eucalyptus trees with bluish leaves exhibit a loss of rooting ability early in their juvenile phase [23]. Comparison of the rooting ability of E. brachyphylla plants at different ages revealed that rooting ability could be detected up to 4 month age, lost at 7 month age, but could be restored by rejuvenation in tissue culture (Figure 2). Concomitantly, miR156 was highly expressed at the base of the above cuttings at 2 month age, after which it declined, whereas miR172 expression was barely detectable in plants younger than 24 months (Figure 2). To restore the rooting ability of mature E. brachyphylla, rejuvenation under tissue-culture conditions was performed (Figure 2). Interestingly, the expression of both miRNAs was very similar in both 7 month age plants and in plantlets that originated from similar plants, and kept in rejuvenation conditions for 24 months for restoration of rooting ability. The data indicate that, rooting ability was not mutually related to a high miR156 expression, and loss of rooting ability was not mutually related to high miR172 expression in the tissue giving rise to ARs. 

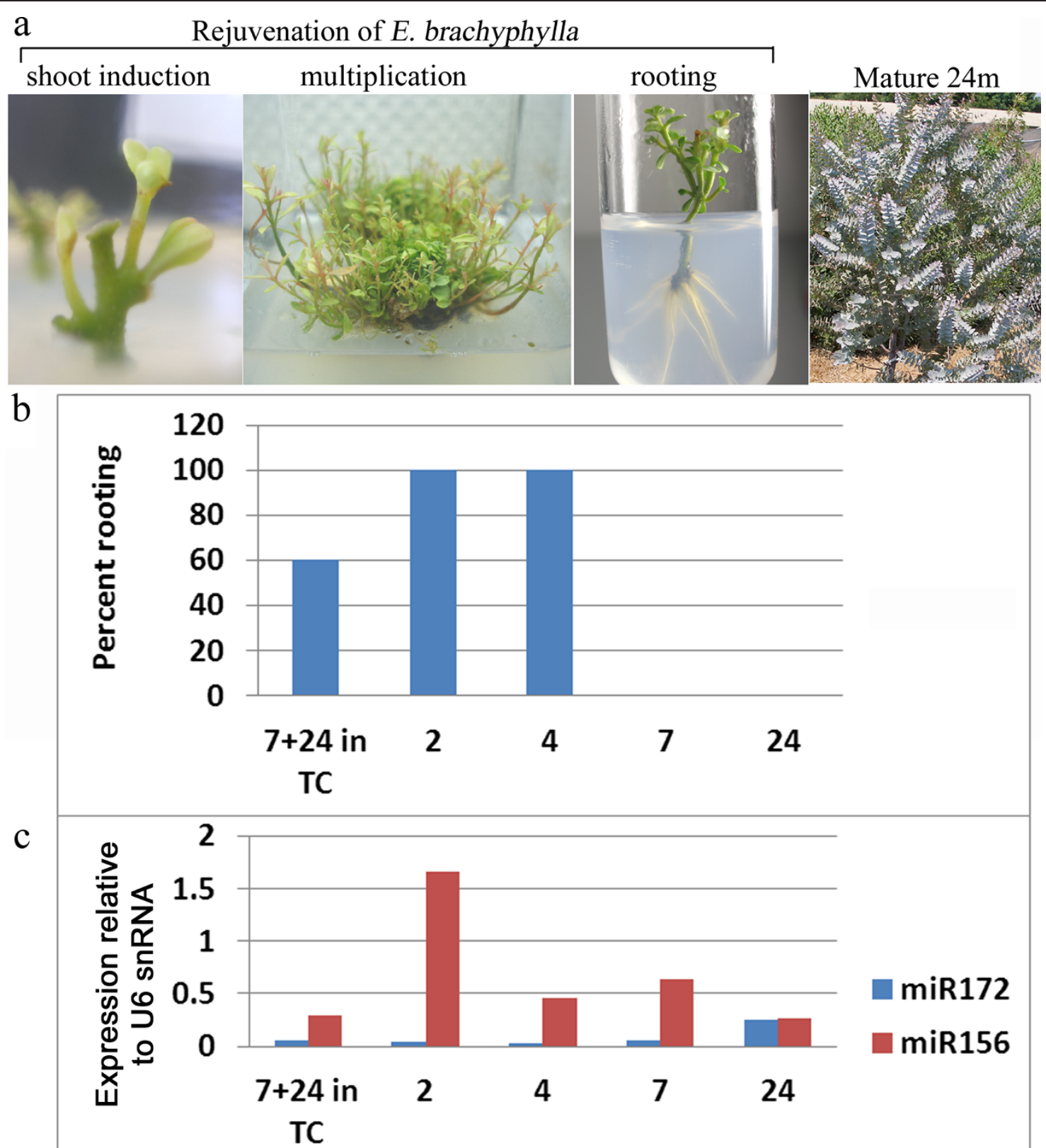

\section{Age of mother plants $(\mathrm{m})$}

d

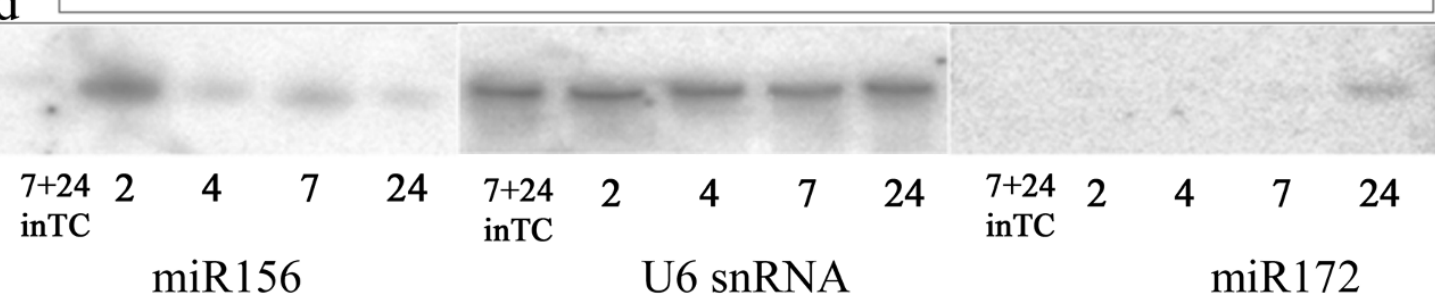

Figure 2 Rejuvenation of $E$. brachyphylla and determination of miR156 and miR172 expression. (a) Sections from a 7-month-old tree, which had lost its rooting ability, rejuvenated in tissue culture (TC). (b) Rooting percentage in E. brachyphylla plants at different ages. (c,d) Analysis of miR156 and miR172 expression: (c) quantitative analysis of the gels in (d). Rooting was determined using 50 cuttings and RNA was prepared from 30 cuttings from each age. 


\section{Profiling the expression of miR156 and miR172 and their predicted targets during induction of AR in $E$. grandis}

To determine the functionality of miR156 and miR172 during the induction of AR formation, their expression was monitored at $0,1,3,6$ and 9 days after rooting was induced. In addition, the expression of a representative predicted target of each of these miRNAs was determined in the same RNA samples (As in Additional file 5: Table S4). In agreement with previous studies $[24,25]$, Figure 3 shows that while the expression of miR156 was higher in juvenile cuttings than in mature ones on day 0, expression of its predicted target EgSPL5 was significantly lower in the juvenile cuttings. Similarly, while the expression of miR172 was lower in juvenile cuttings than in mature ones on day 0 , the expression of its predicted target EgAPETALA 2 was higher in the former, in agreement with previous data [9-11]. Interestingly, miR156 expression was not affected during the first 9 days of induction, whereas that of EgSPL5 in mature cuttings decreased after 1 day and remained low. Similarly, the expression of miR172 in mature cuttings and that of EgAPETALA2 in juvenile cuttings decreased after 1 day and remained low until day 9. These changes led to equalization of the expression of both miRNAs and predicted targets in juvenile and mature cuttings during 3 to 9 days after rooting induction, although only juvenile cuttings formed roots under these conditions.

\section{Discussion}

The change from juvenile to mature phase in plants is a gradual process; culminating in the most recognizable developmental process-flower production. However, prior to the change to the reproductive phase, there is a change from juvenile to adult vegetative phase [2]. The characteristics of this transition are more subtle and differ from one plant species to the other. For example, in Arabidopsis, juvenile characteristics include round and small leaves, lacking trichomes on their abaxial side [26]. The juvenile characteristics in maize include leaf epidermal wax and nodes bearing ARs [7]. It has been shown that miR156 is a regulator of this vegetative phase change in both Arabidopsis and maize. Overexpression of miR156 in Arabidopsis led to increased number of small and round leaves without abaxial trichomes [27]. In maize, it led to increased number of leaves with epidermal wax and nodes with ARs [7,13]. Furthermore, miR156 expression has been found to be correlated with changes in leaf shape in different woody plants [15]. In agreement with previous studies, our observations show that in both $E$. grandis and E. brachyphylla, a relatively high level of miR156 is expressed in juvenile tissues, although no marked changes in leaf shape between the juvenile and mature phases were observed in these Eucalyptus trees, as reported for example for E. globulus [15]. Of note, while the expression of miRNAs was previously determined in the leaves or shoot apices [12,15], we analyzed their expression in the stems. This might explain why we could not relate the timing of the loss of rooting ability to the reduction in miR156 expression. In E. grandis, a significant decline in rooting ability was observed as the distance of the cutting source from the ground increased, although only a subtle decline in miR156 expression was observed in the same plant samples. In E. brachyphylla, a high expression of miR156 was observed in 2-month-old plants. This expression significantly decreased in 4-month-plants and thereafter remained more or less constant in 7 - and 24-month-old plants, as well as in 7-month-old plants that were rejuvenated in tissue culture for 24 months. However, rooting ability was $100 \%$ in 2- and 4-month-old plants, $0 \%$ in 7 - and 24 -month-old plants and $60 \%$ in the rejuvenated plants. The uncoupling of miR156 expression and AR forming ability in these Eucalyptus trees is in agreement with findings obtained from Acacia species. While in Acacia colei the leaf change was correlated to the reduction in miR156 and induction of miR172 [15], in A mangium the leaf change was not correlated to significant differences in rooting abilities [28-31]. Therefore, regulation of AR formation is still unclear. On one hand, cuttings excised close to the root system possess a high rooting potential [2], suggesting the transport of rooting signals from the roots to the stems. On the other hand, overexpression of miR156 in maize Corngrass 1 mutant led to more nodes with ARs [13], and denser aerial roots along the tomato stems [14]. Expression of miR156 also extended the tuber-forming potential to distal axillary buds in potato and tomato [32]. But regulation of miR156 expression in Arabidopsis has been shown to be root-independent and leaf-dependent [33], while, rooting inhibitors have been found in leaves of mature Eucalyptus trees [18]. It might therefore be suggested that the regulation of rooting and the regulation of miR156 are uncoupled, but nevertheless downstream effects of miR156 are relevant for AR formation. The precise relationship between miR156 expression and AR formation in Eucalyptus has yet to be determined.

The expression of miR172 has been shown to be regulated by miR156 via SPL9 and SPL10 [12]. Therefore, the decline in miR156 expression is expected to occur simultaneously with the increase in that of miR172. Indeed, such a correlation has been shown in Arabidopsis [12]. However, a closer look at woody plants revealed a somewhat more complicated picture. When the expression of both miRNAs was analyzed in 1-month-old, and $1-, 4-$, and 10-year-old poplar trees, a gradual decline in miR156 expression was found between 1-month and 4year-old trees, whereas a rise in miR172 expression was only observed in 10-year-old trees, in which there was no further decline in miR156 expression [15]. Similar 


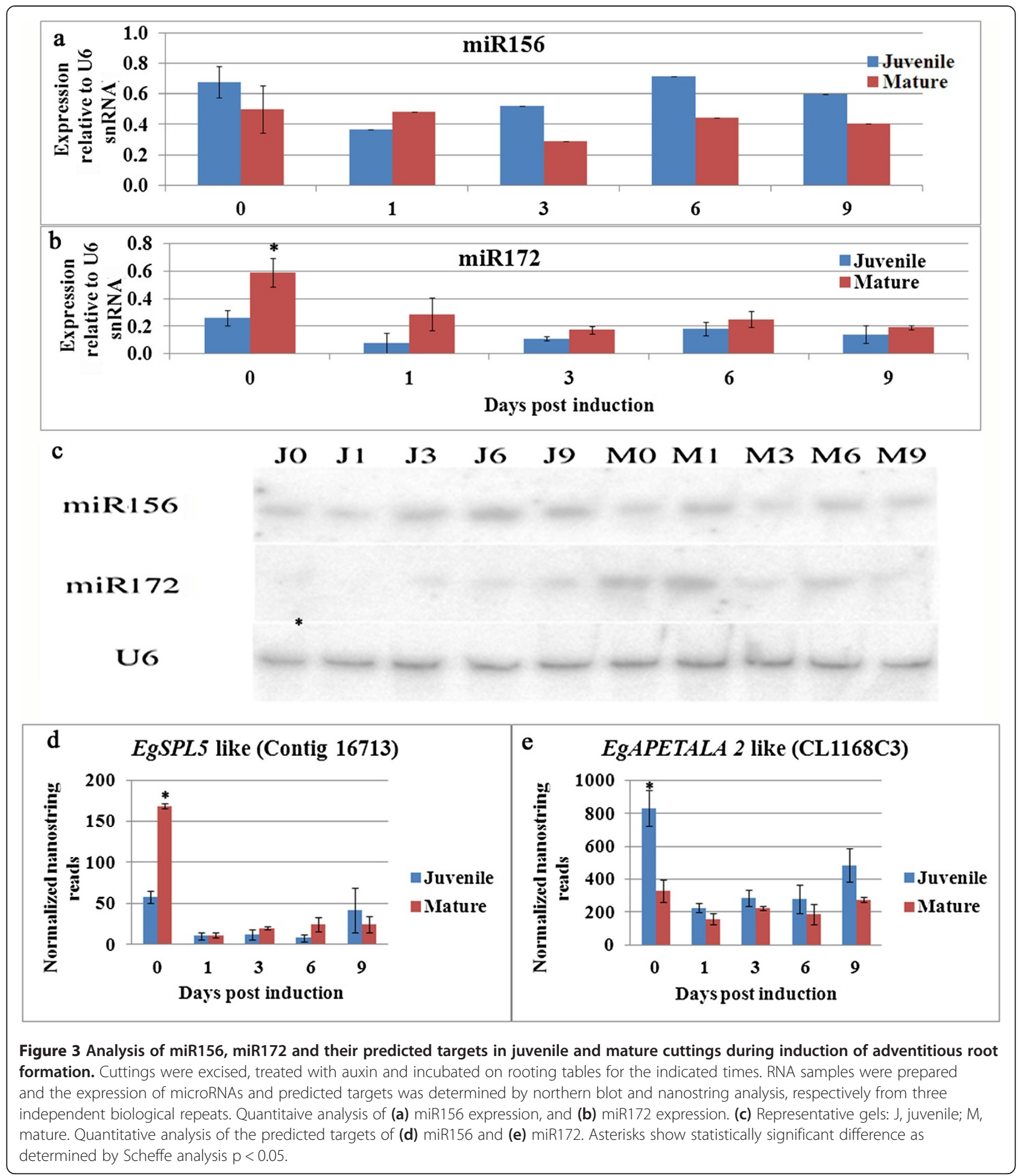

observations were recorded when samples were taken from shoots at different heights $(0.5,2$, and $4 \mathrm{~m})$ or from leaves on the same shoot located at different distances from the main stem. While a gradual decrease in miR156 expression was detected between shoots taken from 0.5 and $2 \mathrm{~m}$ above the ground, no detectable change in miR172 expression was observed in these shoots. An increase in miR172 expression was detected only at 4 $\mathrm{m}$ above the ground. Similarly, when expression was determined in sequential leaves on the same shoot, a 
gradual decrease in miR156 expression was observed with increasing distance from the main stem. However, miR172 expression increased only in the last, most distantly located leaf [15]. Therefore, the decline in miR156 expression preceded the rise in miR172 expression. Our results, albeit obtained from a different tissue, the stems, also show no tight correlation between the timing of miR156 downregulation and miR172 upregulation. Taken together, these data suggest that the expression of miRNA156 and miR172 in woody plants is governed by a complex regulation system, which specifically in cuttings, might be also influenced by wounding, the decrease in leaf area, the loss of roots, and a long rooting period under varying environmental conditions.

\section{Conclusions}

It is concluded that in E. grandis and E. brachyphylla, there is no mutual relationship between easy-to-root cuttings and high miR156 or difficult to root cuttings and high miR172 expression at the base of these cuttings.

\section{Methods \\ Plants}

E. grandis plants were grown from seeds in a nethouse in 15 liter pots containing peat and tuff (70:30, v/v), drip irrigated and fertilized with 3 liters of Shefer737 liquid fertilizer (ICL Fertilizers, Be'er Sheva, Israel) N.P.K $737+$ microelements per $\mathrm{m}^{3}$ of water. E. grandis plants of the same age were hedged at different heights to promote shoot initiation from specific stem sections for cutting production. Cuttings were treated with $6 \mathrm{~g} \mathrm{l}^{-1} \mathrm{~K}$-IBA (Indole-3-butiric acid, potassium salt) for $20 \mathrm{~s}$ and rooted in vermiculite/perlite $1: 1$, heated to $24^{\circ} \mathrm{C}$ under constant $90 \%$ humidity in rooting tables. Rooting was monitored after 30 days. E. brachyphylla cuttings were collected from young seedlings originated from seeds. Seeds were germinated in vermiculite in a temperature-controlled chamber at $23^{\circ} \mathrm{C}$ and transferred to pots at 1 month age. For rooting tests at 2 month age, excised seedlings served as cuttings, whereas at 4 and 7 month age, cuttings were excised from shoots.

To grow E. grandis under sterile conditions, seeds were sterilized in $1 \%$ sodium hypochlorite for $5 \mathrm{~min}$ and washed three times with sterile $\mathrm{ddH}_{2} \mathrm{O}$ before germinating in $10-\mathrm{cm}$ diameter Petri dishes with $0.8 \%$ agar (Sigma) MS (Murashige \& Skoog, Duchefa Biochemie, Haarlem, the Netherlands) growth medium at $24^{\circ} \mathrm{C}$ under a 16-h light/8-h dark cycle. After germination, seedlings were transferred to sterile $50-\mathrm{ml}$ tissue-culture tubes. About 45 days after germination, when the seedlings reached 3 to $4 \mathrm{~cm}$ in height, $\sim 3-\mathrm{cm}$ long cuttings were excised and transferred to a fresh medium. The medium consisted of $0.8 \%$ agar, full-strength Woody Plant Growth medium (Duchefa Biochemie, Haarlem, the Netherlands) containing 3\% (w/v) sucrose, $0.004 \%(\mathrm{w} / \mathrm{v})$ polyvinylpyrrolidone (PVP; Sigma Aldrich), 50 ppm cysteine (Sigma), and $50 \mathrm{ppm}$ ascorbic acid (Sigma); the $\mathrm{pH}$ was adjusted to 5.7 with $\mathrm{KOH}$. Rooting growth medium for cuttings was supplemented with $10 \mu \mathrm{M}$ sodium nitroprusside (Sigma) [34], $5 \mu \mathrm{M} \mathrm{K}-\mathrm{IBA}$, and $8 \mu \mathrm{M}$ auxin conjugate [35].

Rejuvenation of $E$. brachyphylla: To avoid endogenous contamination, mother plants were treated with fungicide ( $2 \mathrm{~g} \mathrm{l}^{-1}$ Daconil) 1 and 2 weeks before the material was introduced into tissue culture. Actively growing branches were immersed in water containing $0.01 \%(\mathrm{v} / \mathrm{v})$ Triton X-100 immediately after cutting, transferred to the lab, and soaked for $1 \mathrm{~h}$ in running tap water. Shoots were cut to about $15 \mathrm{~cm}$ in length, the leaves were removed, and at least half of each pedicle was left attached to the node. Shoots were surface-sterilized with $70 \%(\mathrm{v} / \mathrm{v})$ ethanol for $30 \mathrm{~s}$ and submerged in a solution containing $1.5 \%(\mathrm{v} / \mathrm{v}) \mathrm{NaClO}$ and $0.01 \%$ Triton X-100 with constant agitation for $10 \mathrm{~min}$, followed by four full-volume sterile $\mathrm{H}_{2} \mathrm{O}$ washes. Single nodes were cut from the sterile shoots and induced to form multiple shootlets with the following medium: full-strength McCown Woody Plant Media (Duchefa), $8 \mathrm{~g} \mathrm{l}^{-1}$ agar (Sigma), $30 \mathrm{~g} \mathrm{l}^{-1}$ sucrose, $400 \mathrm{mg} \mathrm{l}^{-1}$ PVP (tissue-culture tested, Sigma), adjusted to pH $5.8 \pm 0.1$ with $1 \mathrm{~N} \mathrm{NaOH}$ before autoclaving. Filter-sterilized ascorbate $\left(50 \mathrm{mg} \mathrm{l}^{-1}\right)$ and cysteine $\left(50 \mathrm{mg} \mathrm{l}^{-1}\right.$ ) were added along with hormones for induction (for hormone types and concentrations see Additional file 6: Table S5). The medium was replaced every 2 to 3 weeks. Growth conditions were a 16 -h photoperiod and, 22 to $25^{\circ} \mathrm{C}$. All hormone stocks were prepared at a concentration of $1 \mathrm{mg} \mathrm{ml}^{-1}(1,000 \mathrm{X})$ and kept at $-20^{\circ} \mathrm{C}$ (up to 6 months). The rejuvenated cultures were maintained by monthly medium replacement for 2 years for gene expression profiling and rooting tests.

\section{RNA preparation}

For deep sequencing, seeds were germinated and plant material samples including roots, stems and leaves were collected 14 days after germination. When cuttings were used, only the $1 \mathrm{~cm}$ lower stem section was taken for RNA extraction. All samples were frozen in liquid nitrogen immediately following collection, ground to a fine powder, and stored at $-80^{\circ} \mathrm{C}$ until use. Total RNA was extracted using Plant/Fungi total RNA purification kit (Norgen Biotek, Thorold, Ontario, Canada) according to the manufacturer's protocol, except that each column was loaded with lysate from $300 \mathrm{mg}$ of plant material instead of $\leq 50 \mathrm{mg}$ to increase RNA yield. Extracted RNA was checked for concentration and purity in an ND1000 spectrophotometer (Thermo Scientific, Wilmington, DE, USA). Samples with a $260 / 280 \mathrm{~nm}$ absorbance ratio of $<1.9$ or $260 / 230 \mathrm{~nm}$ absorbance ratio of $<1.7$ were discarded or further purified with RNeasy 
Mini Kit (Qiagen, www.qiagen.com) according to manufacturer's protocol. RNA samples were kept in the elution buffer at $-80^{\circ} \mathrm{C}$ until use.

\section{Deep sequencing and analysis}

miRNA discovery-oriented deep sequencing was performed by LC Sciences (http://www.lcsciences.com, Houston, TX, USA). Raw sequencing data were processed with the ACGT-miR-v3.5 package (LC Sciences) to filter out adaptors, technical impurities, repetitive sequences, and low-quantity reads $(<3)$ while applying length-cutoff values (15-26 nt), resulting in a nonredundant sequence list. The sequence data was deposited in GEO NCBI (GSE58367, http://www.ncbi.nlm.nih.gov/geo/ query/acc.cgi?acc=GSE58367). The ShortStack small RNA gene annotation tool [19], which analyzes small RNA sequencing data by alignment to a reference genome, was used to infer and annotate small RNA genes.

Target prediction was performed by the psRNATarget tool (http://plantgrn.noble.org/psRNATarget) [36]. To improve the available EST (expressed sequence tags) database of E. grandis, three publicly available E. grandis ESTs were downloaded from The University of Pretoria's Eucalyptus database server (http://eucalyptusdb.bi.up.ac.za/). These included the Kirst.fa with 190,106; leaf_est.fa with 545,604 and e_grandis_xylem_ests_JGI.fa with 218,133 sequences with average length of 163 (nt) and median length of 108 (nt). The sequences were preclustered with TGICL pipeline version 2.1 (http://sourceforge.net/projects/tgicl/) to form similarity groups, from which the CAP3 sequence assembly program [37] was used to assemble 48,397 contigs and 94,070 singletons with average length of 772 (nt) and median length of 706 (nt). BLAST2GO [38] was then used to annotate sequences longer than 300 bases. This was done by Dr. Shifra Ben Dor from the Bioinformatics Unit, Biological Services, Weizmann Institute of Science, Rehovot, Israel. The new database was implemented into the psRNATarget tool.

\section{RNA northern blot analysis}

RNA blot analysis was performed as previously described [39] with several modifications. A polyacrylamide (1:19 bis: acryamide) gel (15\%) (Bio-Rad, https://www.bio-rad.com) was cast in $7 \mathrm{M}$ urea (Sigma) and buffered with $20 \mathrm{mM}$ 4-morpholinepropanesulfonic acid (MOPS; Sigma) using a MiniVE vertical gel system (Huefer, MA, USA) assembled with $9 \times 9 \mathrm{~cm}$ plates and $1.5-\mathrm{mm}$ spacers, and allowed to solidify for $2 \mathrm{~h}$. The gel was then prerun at $100 \mathrm{~V}$ for $15 \mathrm{~min}$ in cold $20 \mathrm{mM}$ MOPS-NaOH ( $\mathrm{pH} 7)$. Deionized formamide (Bio-Rad) was added 1:1 (v/v) to $11 \mu \mathrm{g}$ of total RNA sample, and 6X loading dye (Fermentas, http://www.fermentas.de/) was then added to samples before they were heated to $95^{\circ} \mathrm{C}$ for $2 \mathrm{~min}$ followed by snap cooling on ice. The gel wells were flushed with running buffer using a syringe just prior to sample loading. The gel was run at $100 \mathrm{~V}$ for $2.5 \mathrm{~h}$.

To verify RNA quality on the gel, it was placed in $0.5 \mu \mathrm{g} \mathrm{ml}{ }^{-1}$ fresh ethidium bromide (Hy Laboratories Ltd., Rehovot, Israel), in $20 \mathrm{mM}$ MOPS-NaOH (pH 7) for $10 \mathrm{~min}$ and then transferred onto a UV light box: strongly stained bands of $>60$ nt indicated RNA of acceptable quality. RNA samples were transferred to a Hybond NX nylon membrane (Amersham, http://www. gelifesciences.com) using a Trans-Blot semi-dry electrophoresis system (Bio-Rad) set at $20 \mathrm{~V}$ for $40 \mathrm{~min}$. For cross-linking,the nylon membranes were treated with 0.16 M 1-ethyl-3-(3-dimethylaminopropyl) carbodiimide (EDC; Sigma) intercalating agent in $0.13 \mathrm{M}$ 1-methylimidazole$\mathrm{HCl}(\mathrm{pH} 8)$ at $60^{\circ} \mathrm{C}$ for $60 \mathrm{~min}$. The membranes were then thoroughly rinsed with DEPC (diethylpyrocarbonate)treated water and stored at $-20^{\circ} \mathrm{C}$ until use. Membranes were prehybridized for $1 \mathrm{~h}$ with $15 \mathrm{ml}$ of $\mathrm{EZ}$ hybridization solution (Biological Industries, Beit-Haemek, Israel) at $42^{\circ} \mathrm{C}$. Membranes were then hybridized with 20 pmol oligonucleotides labeled with $20 \mu \mathrm{Ci}$ of $\gamma^{32} \mathrm{P}$ ATP (PerkinElmer, http://www.perkinelmer.com/) at $42^{\circ} \mathrm{C}$ overnight in $\mathrm{EZ}$ hybridization solution. After hybridization, the membrane was washed three times at $55^{\circ} \mathrm{C}$ with $2 \mathrm{X}$ saline sodium citrate (Sigma) and $0.2 \%(\mathrm{w} / \mathrm{v})$ sodium dodecyl sulfate (Sigma) solution for $15 \mathrm{~min}$ and allowed to dry. Autoradiography was performed with FLA-5000 Phosphorimager (Fuji, Tokyo, Japan). Signal intensity of each band was quantified using ImageJ (http://rsb.info. nih.gov/ij/). U6 small nuclear RNA was used to assess the total amount of RNA loaded in each gel well. Each miRNA probe's signal was divided by its corresponding U6 signal.

\section{Expression of predicted target}

Expression analysis of selected predicted targets was performed by Nanostring (www.Nanostring.com, Seattle, WA, USA). The probes used are listed in Additional file 7: Table S6.

\section{Additional files}

Additional file 1: Table S1. E. grandis microRNAs analyzed by ShortStack (numbers separated by commas correspond to each sequence when there is more than one).

Additional file 2: Table S2. Predicted targets of known E. grandis microRNAs.

Additional file 3: Table S3. Predicted targets of $E$. grandis novel microRNAs discovered in this study.

Additional file 4: Figure S1. Levels of expression of microRNAs during adventitious root formation.

Additional file 5: Table S4. Levels of expression of predicted targets during adventitious root formation.

Additional file 6: Table S5. Concentrations of hormones used for tissue culture of E. brachyphylla.

Additional file 7: Table S6. The probes used for the Nanostring analysis. 


\section{Abbreviations}

AR: Adventitious roots; miR: microRNA; IBA: Indole-3- butyric acid.

\section{Competing interests}

The authors declare that they have no competing interests.

\section{Authors' contributions}

$A L, D S, M A-A, I M$ and $Y Y$, performed the experiments, JR, TA and ES designed the experiments, AL and ES wrote the manuscript. All authors read and approved the final manuscript.

\section{Acknowledgment}

We would like to thank Dr. Shifra Ben-Dor from the Bioinformatics Unit, Biological Services, Weizmann Institute of Science, Israel, for building and annotating the E. grandis transcripts. ES acknowledges the support of the KKL (The Israeli Forestry Organization) and the Chief Scientist of the Ministry of Agriculture.

\section{Author details}

${ }^{1}$ The Institute of Plant Sciences, Volcani Center, P.O. Box 6, Bet-Dagan 5025000, Israel. ${ }^{2}$ The Robert H. Smith Institute of Plant Sciences and Genetics in Agriculture, The Robert $\mathrm{H}$. Smith Faculty of Agriculture, Food and Environment, The Hebrew University of Jerusalem, Rehovot 7610001, Israel.

${ }^{3}$ Current address: Syngenta Seeds - R\&D, Valle de Azapa Km 17, Arica, Chile.

Received: 2 April 2014 Accepted: 13 June 2014

Published: 25 June 2014

\section{References}

1. Greenwood MS: Rejuvenation of forest trees. Plant Growth Regul 1987, 6:1-12.

2. Poethig RS: Phase change and the regulation of shoot morphogenesis in plants. Science 1990, 250(4983):923-930

3. Day ME, Greenwood MS, Diaz-Sala C: Age- and size-related trends in woody plant shoot development: regulatory pathways and evidence for genetic control. Tree Physiol 2002, 22(8):507-513.

4. Hackett WP: Juvenility, maturation and rejuvenation in woody plants. Hort Rev 1985, 7:109-155.

5. McGowran E, Douglas GC, Parkinson M: Morphological and physiological markers of juvenility and maturity in shoot cultures of oak (Quercus robur and Q. petraea). Tree Physiol 1998, 18(4):251-257.

6. Poethig RS: The past, present, and future of vegetative phase change. Plant Physiol 2010, 154(2):541-544.

7. Poethig RS: Heterochronic mutations affecting shoot development in maize. Genetics 1988, 119(4):959-973.

8. Evans MM, Passas HJ, Poethig RS: Heterochronic effects of glossy15 mutations on epidermal cell identity in maize. Development 1994 120(7):1971-1981.

9. Moose SP, Sisco PH: Glossy15 controls the epidermal juvenile-to-adult phase transition in maize. Plant Cell 1994, 6(10):1343-1355.

10. Moose SP, Sisco PH: Glossy15, an APETALA2-like gene from maize that regulates leaf epidermal cell identity. Genes Dev 1996, 10(23):3018-3027.

11. Lauter N, Kampani A, Carlson S, Goebel M, Moose SP: microRNA172 downregulates glossy15 to promote vegetative phase change in maize. Proc Natl Acad Sci USA 2005, 102(26):9412-9417.

12. Wu G, Park MY, Conway SR, Wang JW, Weigel D, Poethig RS: The sequential action of miR156 and miR172 regulates developmental timing in Arabidopsis. Cell 2009, 138(4):750-759.

13. Chuck G, Cigan AM, Saeteurn K, Hake S: The heterochronic maize mutant Corngrass 1 results from overexpression of a tandem microRNA. Nat Genet 2007, 39(4):544-549.

14. Zhang X, Zou Z, Zhang J, Zhang Y, Han Q, Hu T, Xu X, Liu H, Li H, Ye Z: FEBS Lett 2010, 585:435-439.

15. Wang JW, Park MY, Wang LJ, Koo Y, Chen XY, Weigel D, Poethig RS: MiRNA Control of Vegetative Phase Change in Trees. PLoS Genet 2011, 7(2):e1002012

16. Gutierrez L, Bussell JD, Pacurar DI, Schwambach J, Pacurar M, Bellini C: Phenotypic plasticity of adventitious rooting in Arabidopsis is controlled by complex regulation of AUXIN RESPONSE FACTOR transcripts and microRNA abundance. Plant Cell 2009, 21(10):3119-3132.

17. Gutierrez L, Mongelard G, Flokova K, Pacurar DI, Novak O, Staswick P, Kowalczyk M, Pacurar M, Demailly H, Geiss G, Bellini C: Auxin Controls
Arabidopsis Adventitious Root Initiation by Regulating Jasmonic Acid Homeostasis. Plant Cell 2012, 24:2515-2527.

18. Paton DM, Willing RR, Nichols W, Pryor LD: Rooting of stem cuttings of Eucalyptus: a rooting inhibitor in adult tissue. Aus J Bot 1970, 18:175-183.

19. Axtell MJ: ShortStack: comprehensive annotation and quantification of small RNA genes. Rna 2013, 19(6):740-751.

20. Ballester A, San-Jose MC, Vidal N, Fernandez-Lorenzo JL, Vieitez AM: Anatomical and biochemical events during in vitro rooting of microcuttings from juvenile and mature phases of chestnut. Ann Bot 1999, 83:619-629.

21. Greenwood MS, Cui X, Xu F: Response to auxin changes during maturation-related loss of adventitious rooting competence in loblolly pine (Pinus taeda) stem cuttings. Physiol Plant 2001, 111(3):373-380.

22. Vidal N, Arellano G, San-Jose MC, Vieitez AM, Ballester A: Developmental stages during the rooting of in-vitro-cultured Quercus robur shoots from material of juvenile and mature origin. Tree Physiol 2003, 23(18):1247-1254.

23. Curir $P$, Vansumere $C F$, Termini A, Barthe $P$, Marchesini A, Dolci $M$ : Flavonoid Accumulation Is Correlated with Adventitious Roots Formation in Eucalyptus gunnii Hook Micropropagated through Axillary Bud Stimulation. Plant Physiol 1990, 92(4):1148-1153.

24. Rhoades MW, Reinhart BJ, Lim LP, Burge CB, Bartel B, Bartel DP: Prediction of plant microRNA targets. Cell 2002, 110(4):513-520.

25. Schwab R, Palatnik JF, Riester M, Schommer C, Schmid M, Weigel D: Specific effects of microRNAs on the plant transcriptome. Dev Cell 2005, 8(4):517-527.

26. Telfer A, Poethig RS: HASTY: a gene that regulates the timing of shoot maturation in Arabidopsis thaliana. Development 1998, 125(10):1889-1898.

27. Wu G, Poethig RS: Temporal regulation of shoot development in Arabidopsis thaliana by miR156 and its target SPL3. Development 2006, 133(18):3539-3547.

28. Monteuuis $\mathrm{O}$ : In vitro rooting of juvenile and mature Acacia mangium microcuttings with reference to leaf morphology as a phase change marker. Trees 2004, 18:77-82

29. Monteuuis $\mathrm{O}$ : In vitro micropropagation and rooting of Acacia magnum microshoots from juvenile and mature origins. In Vitro Cell Dev Biol Plant 2004, 40:102-107.

30. Monteuuis O, Vallauri D, Poupard C, Chauviere M: Rooting Acacia mangium cuttings of different physiological age with reference to leaf morphlogy as a phase change marker. Silvae Genet 1995, 44:150-154.

31. Poupard C, Chauviere M, Monteuuis O: Rooting Acacia mangium cuttings: effects of age, within-shoot position and auxin treatment. Silvae Genet 1994, 43:226-231.

32. Eviatar-Ribak T, Shalit-Kaneh A, Chappell-Maor L, Amsellem Z, Eshed Y, Lifschitz E: A cytokinin-activating enzyme promotes tuber formation in tomato. Curr Biol 2013, 23(12):1057-1064.

33. Yang L, Conway SR, Poethig RS: Vegetative phase change is mediated by a leaf-derived signal that represses the transcription of miR156. Development 2011, 138(2):245-249.

34. Abu-Abied M, Szwerdszarf D, Mordehaev I, Levy A, Stelmakh OR, Belausov E, Yaniv Y, Uliel S, Katzenellenbogen M, Riov J, Ophir R, Sadot E: Microarray analysis revealed upregulation of nitrate reductase in juvenile cuttings of Eucalyptus grandis, which correlated with increased nitric oxide production and adventitious root formation. Plant J 2012, 71:787-799.

35. Riov J: Endogenous and exogenous auxin conjugates in rooting of cuttings. Acta Hortic 1993, 329:284-288.

36. Dai X, Zhao PX: psRNATarget: a plant small RNA target analysis server Nucleic Acids Res 2011, 39(Web Server issue):W155-159.

37. Huang $X$, Madan A: CAP3: A DNA sequence assembly program. Genome Res 1999, 9(9):868-877.

38. Conesa A, Gotz S, Garcia-Gomez JM, Terol J, Talon M, Robles M: Blast2GO: a universal tool for annotation, visualization and analysis in functional genomics research. Bioinformatics 2005, 21(18):3674-3676.

39. Pall GS, Hamilton AJ: Improved northern blot method for enhanced detection of small RNA. Nat Protoc 2008, 3(6):1077-1084

doi:10.1186/1471-2164-15-524

Cite this article as: Levy et al.: Profiling microRNAs in Eucalyptus grandis reveals no mutual relationship between alterations in miR156 and miR172 expression and adventitious root induction during development. BMC Genomics 2014 15:524. 